\title{
Personal Protective Equipment
}

\section{Priya Sharma}

Practice Management Consultant (Compliance), British Dental Association

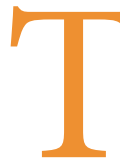

he pandemic brought to the forefront the use of PPE in dental practices and as a result, an evolution regarding its use in dentistry. Here we explore some of those changes.

\section{Aerosol Generating Procedures (AGPs)}

Whilst undertaking AGPs the following PPE is recommended:

$\rightarrow$ A long-sleeved disposable fluid repellent gown (covering the arms and body) or

$\rightarrow$ disposable fluid repellent coveralls

$\rightarrow$ A filtering face piece class 3 (FFP3) respirator

$\rightarrow$ Eye/face protection (visors)

$\rightarrow$ Gloves.

\section{Non-Aerosol Generating Procedures} (non-AGPs)

Generally, PPE that was worn pre-pandemic is recommended while undertaking non-AGP; this would include FRSM (fluid resistant surgical mask), eye protection and gloves. A disposable plastic apron is also suggested.

\section{Respiratory Protective Equipment (RPE)} As the name suggests, RPE is used to protect the dental professional against the inhalation of hazardous substances. A respirator protects the wearer while a surgical mask is not designed to protect the wearer although may prevent larger droplets from penetrating the mask. Non-valved respirators are preferred as there is filtration of the exhaled air by the wearer. A valved respirator does not protect patients. There is no respirator which will be $100 \%$ effective.

\section{Fit testing}

Fit testing is a requirement for FFP3 masks, this ensures that the specific make and model matches the facial features and creates an adequate seal in turn offering maximum protection. Repeat fit testing will be required for different makes/models of FFP3 masks or if there have been substantial changes in facial features. Public Health England (PHE) suggests FFP3 masks may be considered if all manufacturer's instructions are followed.

\section{Powered air purifying respirator (PAPR)}

Where a FFP3 mask is not suitable consideration may be given to a PAPR. These do not require fit testing. Various clinical aids may pose a difficulty under the PAPR to wearers. There may also be suitability problems whilst wearing religious articles of faith. They weight approximately $2 \mathrm{~kg}$ and cost anywhere between $£ 500$ to $£ 1200$.

\section{Donning PPE}

The dental team must be aware of appropriate donning and doffing technique. The following instructions may be followed for donning PPE for an AGP:

$\rightarrow$ Remove jewellery/watch

$\rightarrow$ Tie long hair back

$\rightarrow$ Perform hand hygiene

$\rightarrow$ Put on the long-sleeved fluid repellent gown

$\rightarrow$ Fit the respirator, ensure adequate seal has been created

$\rightarrow$ Put on your full face protection/visor

$\rightarrow$ Put on your disposable gloves and cover the cuffs of your gown.

Ideally, PPE should be donned in a separate room to the surgery. If this is not possible then PPE may be donned prior to the patient's arrival in the clean surgery.

\section{Doffing PPE}

Generally, PPE should be doffed in the reverse order in which one has donned the PPE. Care should be exercised to ensure that contaminated surfaces of PPE do not come into contact with unprotected parts of the body.

The following technique is recommended:

$\rightarrow$ Remove gloves

$\rightarrow$ Clean hands with alcohol based hand sanitiser

$\rightarrow$ Remove gown, being mindful that the front of the gown and sleeves will be contaminated

$\rightarrow$ Remove face protection

$\rightarrow$ Clean hands with alcohol based hand sanitiser

$\rightarrow$ Remove respirator ensuring not to touch contaminated surfaces

$\rightarrow$ Perform hand hygiene.
Vigilance should be used in doffing PPE ensuring cross contamination potential is minimised. All PPE may be removed in surgery except for face protection and respirator which should be removed in a separate room.

\section{Sessional use of PPE}

There remains some confusion in the dental community regarding the sessional use of PPE. A single session refers to a specific period of time where a dental professional is undertaking duties in a specific clinical care setting or exposure environment. The session would deem to have ended when the clinical provider leaves the clinical setting or exposure environment. Respirators, masks, eye protection and disposable fluid repellent coveralls or longsleeved disposable fluid repellent gowns can be used for a single session if they are still fit for purpose. Aprons and gloves are single use PPE and should be disposed of safely.

\section{Challenges regarding PPE}

One of of the continual challenges we face in a wide-range of matters regarding PPE would be shortages. Initially, there was also a flooding of the market with counterfeit PPE partnered with the fact that desperately dental professionals were procuring PPE from nondental suppliers. Followed by the unreasonable escalation in costs in acquiring PPE. Until 20 February 2021 NHS practices in England could claim for PPE reimbursement for all COVID-19 PPE used in the provision of NHS dental services between 27 February 2020 and 31 December 2020.

\section{Future of PPE}

We have come a long way in a year's time in understanding coronavirus along with the required infection control requirements. The main transmission modalities of coronavirus are respiratory and airborne, as such dental professionals are at a higher risk of contracting the virus because their clinical work is in such close proximity to the potential source of the virus. Even if dental team members have received one dose of the COVID-19 vaccine, adequate PPE must still be continued to be worn to interrupt the transmission of COVID-19. https://doi.org/10.1038/s41404-021-0681-z 